\title{
Las Redes Sociales de Internet para la promoción de pueblos turísticos.
}

\section{Internet's Social Media in the promotion of touristic towns.}

\author{
Lic. Massiel Martínez Carballo., ${ }^{1}$ Msc. Martha Omara Robert Beatón., ${ }^{2}$ \& DraC. Lisandra Torres \\ Hechavarría. $^{3}$
}

\section{Resumen.}

Las redes sociales de Internet se tornan un medio altamente efectivo para la comunicación de atractivos turísticos, partiendo de la idea de que una empresa o destino turístico está presente hoy en una red social, aunque la directiva de los mismos esté consciente de ello o no, le confiere especial importancia. El presente trabajo persiguió como objetivo proponer acciones para la gestión de las redes sociales de Internet en los pueblos turísticos cubanos. Se aplicaron métodos y técnicas como: la revisión bibliográfica, el inductivodeductivo y análisis-síntesis, entrevistas, así como un benchmarking en función del comportamiento de las redes sociales de Internet en tres pueblos turísticos: Puebla, Obermutten y Morella, lo cual permitió un acercamiento al alcance y el empleo de las mismas, al mismo tiempo que contribuyó a la propuesta de acciones encaminadas a su gestión en pueblos turísticos cubanos.

Palabras claves: Redes Sociales, Internet, Pueblos Turísticos.

\section{Abstract.}

The Internet's Social Media have become a highly effective mean for the communication of touristic assets, based on the idea that a company or touristic destination has presence today in a social media, whether their managers are aware of it or no. The following work's primary objective is to propose actions for the management of Internet's Social Media in touristic Cuban small towns. During the investigation methods and techniques like bibliographic checkup, inductive-deductive and analysis-synthesis, interviews, were applied. Also a benchmarking analysis was developed based in the performance of Internet's Social Media in three touristic towns: Puebla, Obermutten and Morella this benchmarking allowed a closer insight look to the reach and employment of these Social

\footnotetext{
${ }^{1}$ Universidad de la Habana, Facultad de Turismo. La Habana, Cuba. massiel.martinez@ftur.uh.cu

${ }^{2}$ Universidad de la Habana, Facultad de Turismo. La Habana, Cuba. omara_robert@ftur.uh.cu

${ }^{3}$ Universidad de la Habana, Facultad de Turismo. La Habana, Cuba. lisandra_torres@ftur.uh.cu
} 
Medias and at the same time it allowed the proposal of a set of actions aimed at their management in Cuban touristic towns.

Keywords: Social Media, Internet, touristic towns

\section{Introducción}

Vivimos en una era informatizada donde prácticamente no se concibe el desarrollo diario sin acudir en algún momento a la utilización de un medio tecnológico. Las distancias se acortan a décimas de segundo gracias a un ordenador, tablet o Smartphone y son pocos los negocios exitosos, independientemente del sector al que pertenezcan, los que se conciben sin la utilización de las facilidades de Internet.

La comunicación promocional no quedó ajena a la revolución que constituyó la generalización del uso de Ia Red de Redes, no es necesario contar con un gran grupo de personas recorriendo el país para mostrar los productos en todo su detalle y llegar al potencial comprador. Ni la radio ni la televisión y mucho menos la prensa podía llegar a donde llegaba Internet, y si el cliente decide comprar basta un simple clic [1].

Según las Estadísticas 2017 de Internet World Stads, 3885 millones de personas son usuarios de Internet, lo que representa más de la mitad de la población mundial y de ellos 2 196millones son usuarios de Facebook. Tradicionalmente los internautas se habían concentrado en los países del llamado primer mundo, pero en la última década se ha dado una expansión acelerada y considerable del acceso a Internet en regiones del mundo aún en vías de desarrollo, como Latinoamérica, África y Asia. No obstante a los esfuerzos del gobierno cubano para incrementar la conectividad a la red de redes, tarea a la cual se la he dado especial prioridad; aun presenta desventajas con el desarrollo que se ha alcanzado a nivel mundial con el uso de las tecnologías de la informática y las comunicaciones reflejado en la poca utilización de las tecnologías como principal herramienta de gestión para la consecución de objetivos de las diferentes instituciones o entidades del sector turístico. Elemento al que se debe especial interés si se tiene en cuenta que los principales mercados emisores de turistas a la Isla se encuentran en la vanguardia en el uso del mismo. De acuerdo con el Google Consumer Barometer el 89\% de los canadienses poseen acceso a Internet y de ellos el $79 \%$ accede diariamente y también es conocido su amplia aplicación por parte de los principales competidores. Por tanto avanzar en la materia del uso de Internet, es un reto de vital importancia para el desarrollo de un turismo acorde a las tendencias del mercado contemporáneo.

Un pueblo turístico es un espacio geográfico con atractivos turísticos donde está enclavada una comunidad o asentamiento poblacional, reordenado para introducir y desarrollar un turismo responsable con la conservación del medio ambiente, las tradiciones, los valores sociales y comunitarios, con una concepción urbanística y estilo arquitectónico integrados al contexto local e histórico, introduciendo tecnologías ecológicas, autosuficiente en su uso mixto turístico-comunidad [2;3].

Variados son los ejemplos a nivel mundial de pueblos turísticos que se encuentran en la vanguardia del empleo de Internet, y específicamente de sus Redes Sociales para la generación de ventajas competitivas, traducidas en mayor cantidad de clientes e ingresos turísticos, ¿en qué posición se encuentran los pueblos turísticos cubanos y qué pasos deberían darse para acercarse a las tendencias internacionales? 
El objetivo general de este trabajo es proponer acciones para la gestión de las Redes Sociales de Internet en los pueblos turísticos cubanos, para dar cumplimiento al mismo se trazaron como objetivos específicos identificar el alcance de las redes sociales de Internet, exponer la actualidad cubana con respecto la conectividad e infraestructura para la navegación en Internet y analizar la gestión de redes sociales de Internet en pueblos turísticos exitosos a escala global.

La investigación contribuye a la creación de una mayor conciencia sobre la necesidad de trabajar en un empleo coordinado y coherente de las redes sociales de Internet, ya constituyen una herramienta de comunicación y marketing muy poderosa y su alcance se encuentra subestimado en el sector turístico cubano. La misma tributa alo expresado en el Lineamiento 211 del VII Congreso del Partido Comunista de Cuba: Perfeccionar las formas de comercialización, utilizando las tecnologías más avanzadas de la información y la comunicación, y potenciando la comunicación promocional [4].

\section{Métodos:}

La presente investigación es de tipo descriptiva, ya que en el desarrollo del estudio científico se describe lo más preciso posible el fenómeno que se investiga [5].La información recopilada mediante la revisión y el análisis bibliográfico del tema objeto de estudio, permitió la elaboración de las bases teóricas de la Investigación. Se realizó una búsqueda en Internet, para identificar la presencia de los pueblos turísticos cubanos, contrastándola con un Benchmarking a los pueblos turísticos: Puebla, Obermutten y Morella, los que fueron seleccionados por haber demostrado ser exitosos en la gestión de las redes sociales, presentar características diferentes entre sí, acorde a las modalidades turísticas desarrolladas y formas de gestión. El análisis partió del empleo de los indicadores de medición de eficiencia para redes sociales de Internet definidos por Quesada (2016): número de personas a las que llegan las publicaciones, número de fans o seguidores, suscriptores, visitas, comentarios, reproducciones, clics en el botón me gusta (Likes), número de veces que se comparte una publicación, periodicidad de las publicaciones y generación de contenido [6]. Otra técnica de recopilación de información que aportó datos interesantes fueron las entrevistas no estructuradas a los community managers de las páginas de Facebook de Obermutten y de Morella quienes ofrecieron datos interesantes desde su experiencia para la gestión de redes sociales. A partir de los resultados del Benchmarking unido con las características propias de la conectividad a Internet en Cuba se propusieron acciones para gestionar las Redes sociales de Internet seleccionadas. A lo largo de la investigación se utilizaron los métodos histórico-lógico, análisis y síntesis e inductivo-deductivo.

\section{Resultados.}

Se seleccionaron las Redes Sociales de Internet afines a la temática de la presente investigación, las cuales se muestran en la tabla 1. En el año 2010 existía un total de 1.22 miles de millones de usuarios de Redes Sociales y ya en el año 2017 esta cifra era de 2.44 miles de millones de usuarios (Statista 2018).

Tabla 1:Número de usuarios en 2018 en Redes Sociales de Internet. 


\begin{tabular}{|l|l|}
\hline Red Social de Internet & $\begin{array}{l}\text { Usuarios en iulio de 2018 } \\
\text { (cifras en millones) }\end{array}$ \\
\hline Facebook & $\mathbf{2 1 9 6}$ \\
\hline YouTube & $\mathbf{1 9 0 0}$ \\
\hline WhatsApp & $\mathbf{1 5 0 0}$ \\
\hline Instagram & $\mathbf{1 0 0 0}$ \\
\hline Sina Weibo & $\mathbf{4 1 1}$ \\
\hline Twitter & $\mathbf{3 3 6}$ \\
\hline LinkedIn & $\mathbf{2 9 4}$ \\
\hline Pinterest & $\mathbf{2 0 0}$ \\
\hline
\end{tabular}

Fuente: Elaboración Propia datos Obtenidos de Statista 2018.

Situación actual del acceso a Internet en Cuba y peculiaridades de los pueblos turísticos.

En el año 2017, se crearon 395 nuevas áreas de navegación; 46 salas de navegación alámbricas y 349 espacios para la navegación Wifi (278 localizadas en sitios públicos) Actualmente la Isla cuenta con 1552 plazas para la navegación, de ellas 595 Wifi de acceso público en todos sus municipios y la posibilidad de 125 mil 460 conexiones simultáneas, 202 salas de navegación de ETECSA y 755 áreas de navegación de terceros. Con la implementación del servicio Nauta Hogar el 29 de septiembre de 2017, se logró, al cierre del año llegar a 14 mil 634 hogares, ubicados en 103 municipios y 256 Consejos Populares [7].

Los pueblos turísticos cubanos en su mayoría cuentan con una infraestructura de conexión a Internet limitada, algunos como San Miguel de los Baños no cuentan tan siquiera con uno de los conocidos "parques Wifi", así mismo existen carencias en cuanto a la formación del personal en temas relativos a la gestión de las redes sociales como instrumento para la promoción de valores turísticos.

\section{El empleo de Redes Sociales de Internet en la promoción de Pueblos Turísticos del Mundo.}

El caso Puebla: El sitio web oficial, "Puebla es mi destino" posee enlaces con las redes sociales: Facebook, Twitter, YouTube e Instagram.

\section{Puebla en YouTube.}

$\checkmark$ Canal Oficial: Puebla Travel

$\checkmark$ Subscriptores: 98

$\checkmark$ Vistas:12 414

$\checkmark$ Descripción: Canal oficial de la Secretaría de Cultura y Turismo del gobierno del estado de Puebla dedicado a la promoción cultural y turística del estado de Puebla.

$\checkmark$ Links: Puebla.Travel, Facebook, Twitter, Instagram.

$\checkmark 20$ Videos. 
$\checkmark$ Video con más vistas: Tehuacán un lugar lleno de historia. (2852 vistas). Publicado en Marzo 29, 2018.

$\checkmark$ Periodicidad entre videos: Aproximadamente 2 meses.

$\checkmark$ URL: https://www.youtube.com/pueblatravel/.

Tabla 2: Análisis de la Página de Facebook de Puebla.

\begin{tabular}{|l|l|l|l|}
\hline URL & $\begin{array}{l}\text { Enlace con el Sitio Web } \\
\text { Oficial }\end{array}$ & $\begin{array}{l}\text { \# de Likes de la } \\
\text { página }\end{array}$ & de Seguidores \\
\hline https://www.facebook.com/PueblaTravel & $\begin{array}{l}\text { Sí } \\
\text { (www.puebla.travel) }\end{array}$ & 518091 & 514466 \\
\hline Tipo de Contenido & $\begin{array}{l}\text { Periodicidad de las } \\
\text { Publicaciones }\end{array}$ & $\begin{array}{l}\text { Likes por } \\
\text { Publicación }\end{array}$ & $\begin{array}{l}\text { Comentarios Publicaciones } \\
\text { por }\end{array}$ \\
\hline $\begin{array}{l}\text { Imágenes } \\
\text { Videos }\end{array}$ & $\begin{array}{l}\text { Aproximadamente } \\
\text { veces al día. }\end{array}$ & $\begin{array}{l}\text { Oscilan entre los } \\
150 \text { y los 200 } \\
\text { likes. }\end{array}$ & $\begin{array}{l}\text { Oscilan entre los } 12 \text { comentarios. } \\
\text { Artículos }\end{array}$ \\
\hline Publicaciones Compartidas & Otras informaciones \\
\hline $\begin{array}{l}\text { Todas las publicaciones son compartidas. } \\
\text { Oscilando entre las 2 y las 25 veces, } \\
\text { existiendo publicaciones que han llegado } \\
\text { a ser compartidas hasta 115 veces. }\end{array}$ & $\begin{array}{l}\text { La página permanece actualizada, dando promoción a los } \\
\text { principales eventos que se realizan. } \\
\text { Los videos gozan de gran aceptación siendo reproducidos } \\
\text { algunos hasta 3 mil veces. } \\
\text { Se utiliza la transmisión en vivo. } \\
\text { Pocos comentarios y no son respondidos por los } \\
\text { administradores. }\end{array}$ \\
\hline
\end{tabular}

Fuente: Elaboración Propia. Datos obtenidos de la Página de Facebook de Puebla (abril 2018).

Puebla en Twitter: @Puebla (https://twitter.com/puebla)se unió a Twitter en septiembre de 2011. Desde ese entonces ha subido un total de 11700 fotos y videos. Posee un total de 29000 tweets, se encuentra siguiendo a 1373 otras cuentas y posee un total de 49800 seguidores, y 833 “Me gusta”. Posee enlaces con Facebook y el sitio web Puebla.travel. Es la cuenta oficial de promoción de la secretaría de cultura y turismo del estado de Puebla. Realiza aproximadamente dos publicaciones diarias, se anuncian los principales eventos a realizar: ferias, conciertos exposiciones. Además de esta cuenta, existen otras que se dedican a promocionar el estado de Puebla, como son: @VisitaPuebla, @PueblaMuseos, @IMACP (arte y cultura Puebla), @FeriaPuebla.

Puebla en Instagram: Cuenta oficial de promoción turística de la Secretaría de Turismo del estado de Puebla. (https://www.instagram.com/pueblatravel/) un total de 269 publicaciones, se encuentra siguiendo a 120 otras cuentas y posee un total de 51800 seguidores. Las fotografías en su mayoría sobrepasan los 900 likes, llegando a sobrepasar la cifra de los 2000, los comentarios oscilan entre los 10 y 100 . Se publican fotografías diariamente, las cuales promocionan atractivos históricos, culturales, gastronómicos, actividades, concursos del lugar.

El caso Obermutten: El sitio web oficial www.mutten.ch, solo cuenta con enlace a su página de Facebook y es aquí donde se centran todos los esfuerzos de su promoción en Redes sociales [8]. 
Tabla 3: Análisis de la Página de Facebook de Obermutten.

\begin{tabular}{|c|c|c|c|}
\hline URL & $\begin{array}{l}\text { Enlace con el Sitio Web } \\
\text { Oficial }\end{array}$ & $\begin{array}{l}\text { \# de Likes de la } \\
\text { página }\end{array}$ & \# de Seguidores \\
\hline https://www.facebook.com/obermutten/ & $\begin{array}{l}\text { Sí } \\
\text { www.mutten.ch }\end{array}$ & 38077 & 36080 \\
\hline Tipo de Contenido & $\begin{array}{l}\text { Periodicidad de las } \\
\text { Publicaciones }\end{array}$ & $\begin{array}{ll}\text { Likes } & \text { por } \\
\text { Publicación } & \end{array}$ & $\begin{array}{ll}\text { Comentarios } & \text { por } \\
\text { Publicaciones } & \end{array}$ \\
\hline $\begin{array}{l}\text { Imágenes } \\
\text { Videos } \\
\text { Artículos }\end{array}$ & $\begin{array}{l}\text { Aproximadamente } \\
\text { publicación semanal }\end{array}$ & $\begin{array}{l}\text { Oscila entre los } 30 \\
\text { y } 150 \text { likes }\end{array}$ & $\begin{array}{l}\text { Oscila entre los } 5 \text { y } 10 \\
\text { comentarios. }\end{array}$ \\
\hline Publicaciones Compartidas & \multicolumn{3}{|l|}{ Otras informaciones } \\
\hline $\begin{array}{l}\text { La mayoría de las publicaciones son } \\
\text { compartidas, desde } 1 \text { hasta } 40 \text { veces } \\
\text { aproximadamente, }\end{array}$ & \multicolumn{3}{|c|}{$\begin{array}{cl}\checkmark & \text { La página permanece actualizada. } \\
\checkmark & \text { Los videos cuentan con gran aceptación siendo reproducidos } \\
& \text { hasta } 3000 \text { veces en promedio. } \\
\checkmark & \text { Existe interacción entre el administrador de la página y los } \\
& \text { usuarios ya que todos los comentarios son respondidos. } \\
\checkmark & \text { Se utiliza la transmisión en vivo. } \\
\checkmark & \text { Se anuncian concursos en la página } \\
\checkmark & \text { Calificación por los usuarios 4.9 estrellas }\end{array}$} \\
\hline
\end{tabular}

Fuente: Elaboración Propia. Datos obtenidos de la Página de Facebook de Obermutten (abril 2018)

El caso Morella: El sitio web oficial de Morella, "Morella Turística" (http://www.morellaturistica.com) posee enlaces con las redes sociales: Facebook y Twitter.

Tabla 4:Análisis de la Página de Facebook de Morella

\begin{tabular}{|c|c|c|c|}
\hline URL & $\begin{array}{l}\text { Enlace con el Sitio Web } \\
\text { Oficial }\end{array}$ & $\begin{array}{l}\text { \# de Likes de } \\
\text { la página }\end{array}$ & \# de Seguidores \\
\hline https://www.facebook.com/morellaturistica & $\begin{array}{l}\text { Sí } \\
\text { www.morellaturistica.com }\end{array}$ & 8.030 & 7.997 \\
\hline Tipo de Contenido & $\begin{array}{l}\text { Periodicidad de las } \\
\text { Publicaciones }\end{array}$ & $\begin{array}{l}\text { Likes por } \\
\text { Publicación }\end{array}$ & $\begin{array}{l}\text { Comentarios por } \\
\text { Publicaciones }\end{array}$ \\
\hline $\begin{array}{l}\text { Imágenes } \\
\text { Videos } \\
\text { Artículos }\end{array}$ & $\begin{array}{l}\text { Aproximadamente } \\
\text { publicación diaria. }\end{array}$ & $\begin{array}{l}\text { Oscilan entre } \\
\text { los } 100 \text { y los } 20 \\
\text { likes. Aunque } \\
\text { hay } \\
\text { publicaciones } \\
\text { que alcanzan } \\
\text { los } 500 \text { likes }\end{array}$ & $\begin{array}{lr}\text { Oscilan entre los } 2 \\
\mathrm{y} \quad \text { los } \\
\text { comentarios. }\end{array}$ \\
\hline Publicaciones Compartidas & \multicolumn{3}{|l|}{ Otras informaciones } \\
\hline $\begin{array}{l}\text { Todas las publicaciones son compartidas. } \\
\text { Oscilando entre las } 1 \text { y las } 7 \text { veces. }\end{array}$ & \multicolumn{3}{|c|}{$\begin{aligned} \checkmark & \text { La página permanece actualizada, dando promoción a los } \\
& \text { principales eventos que se realizan. } \\
\checkmark & \text { Los videos gozan de gran aceptación siendo reproducidos } \\
& \text { algunos hasta } 6 \text { mil veces. } \\
\checkmark & \text { Se utiliza la transmisión en vivo. } \\
\checkmark & \text { Pocos comentarios y no son respondidos por los } \\
& \text { administradores. } \\
\checkmark & \text { Se promocionan eventos del pueblo. } \\
\checkmark & \text { Calificación por los usuarios } 4.8 \text { de } 5 \text { estrellas. }\end{aligned}$} \\
\hline
\end{tabular}


Fuente: Elaboración Propia. Datos obtenidos de la Página de Facebook de Morella (abril 2018)

@morellaturismo (https://twitter.com/MorellaTurística) Se unió a Twitter en noviembre de 2010. Desde ese entonces ha subido un total de 567 fotos y videos. Posee un total de 4107 tweets, se encuentra siguiendo a 83 otras cuentas y posee un total de 3 392 seguidores, y 1079 "Me gusta". Posee enlaces con el sitio web morellaturistica.com. Realiza aproximadamente una publicación diaria, se anuncian los principales eventos a realizar: ferias, actividades deportivas, concursos, exposiciones, etc.

\section{Los pueblos turísticos cubanos, propuesta de acciones para su comunicación mediante redes sociales de Internet.}

Al realizar una búsqueda en Internet sobre diferentes pueblos turísticos cubanos, se pudo comprobar que los mismos aparecen pero su comunicación ocurre por medio de actores diversos, quienes emplean su imagen atendiendo a intereses propios. Para lograr una gestión coordinada y consciente de la presencia de los pueblos turísticos cubanos en Internet se proponen las siguientes acciones:

- Habilitar la infraestructura mínima necesaria para la conexión a internet en los pueblos turísticos.

- Capacitar a los residentes en temas relativos a la comunicación promocional y mediante Internet.

- Autogestionar su comunicación coordinada y conscientemente desde la comunidad.

- Definir puestos de trabajo Comunity Manager y Social Media Manager de acueredo con las funciones planteadas por Ortega (2017) [9].

- Crear un sitio web (no debe ser únicamente turístico sino que también se pueden ofrecer otras informaciones de interés, tales como: ubicación, clima, moneda, qué hacer en el pueblo, noticias destacadas. Poseerá enlaces con las redes sociales)

- Crear perfiles o páginas en los Redes Sociales Facebook, Twitter e Instagram.

- Ubicar enlaces desde el sitio Web hacia las Redes Sociales y viceversa.

- Generar contenido (principales atractivos, historia, noticias, acontecimientos relevantes, valores autóctonos de la comunidad).

- Realizar Marketing de Influencers[10].

- Crear un logo que identifique al pueblo.

- Emplear las etiquetas populares de cada red social.

- Ubicar enlaces del sitio web en el sitio web Cuba Travel.

- Colocar los enlaces del sitio web y las redes sociales para promocionar en otros ámbitos y de forma offline.

- Monitorear constantemente el sitio web y las redes sociales mediante los indicadores de medición de eficiencia. 
- Negociar con las cadenas hoteleras que operan en el destino Cuba y que cuentan con reconocimiento internacional y estrategias de comunicación online bien gestionadas para que formen parte de su promoción.[11].

\section{Discusión}

Las redes sociales de Internet han mostrado un crecimiento acelerado, constituyendo un elemento clave de la comunicación en el siglo XXI. Ocupan un lugar privilegiado para las empresas turísticas, ya que, al momento de elegir un destino para vacacionar, los usuarios buscan a través de estas, hoteles, aerolíneas, tiendas, cines, museos, centros comerciales, restaurantes y transporte; propiciando el llamado fenómeno de los viajeros 2.0, reconocido como el turista que recomienda, comparte en tiempo real: fotografías, sentimientos, información y experiencias relevantes, tanto positivas como negativas de los servicios turísticos y sus destinos[12].

Existe un incremento en la conectividad del país (Cuba) a partir del 2015, no obstante aún resulta insuficiente. En los pueblos turísticos es necesario aumentar no solamente en infraestructura, sino también en conciencia y aplicar los recursos ya existentes en una gestión que permita aprovechar las ventajas que ofrece el Internet y sus Redes Sociales, lo cual sería un paso de avance en la anhelada gestión desde las comunidades. Las redes sociales de internet permiten acceder a públicos objetivos, como inversores potenciales, los cuales podrían contribuir al rescate de los recursos de muchos de los pueblos turísticos cubanos.

Se puede constatar que los tres pueblos turísticos seleccionados emplean Facebook para su promoción, esto podría justificarse con el hecho de que esta red social es la que posee a escala global un mayor número de usuarios y permite la transmisión de contenido de valor. En el caso de Facebook, Twitter e Instagram permanecen actualizados diariamente, al menos con una publicación. El número de subscriptores o seguidores de las Redes sociales empleadas por los pueblos turísticos, sobrepasa el número de residentes. Se promocionan las actividades que se realizan en los pueblos, como: concursos, conciertos, ferias, exposiciones y actividades deportivo- recreativas. En el caso de YouTube, solamente es empleado en la actualidad por Puebla, esta red social es la segunda con mayor alcance a escala global, sin embargo la producción de videos promocionales es costosa, por lo que la periodicidad de los videos es de 2 meses aproximadamente. Las Redes sociales de los tres pueblos turísticos son gestionadas de forma consciente, generando contenido atractivo para los usuarios lo cual es reflejado en el engagement de los mismos. Todas cuentan con enlaces al sitio web del destino, donde se concretan las ventas de alojamiento, excursiones y experiencias.

Las acciones propuestas se encuentran ajustadas a la realidad de los pueblos turísticos cubanos y se nutren de la experiencia de competidores mundiales.

\section{Conclusiones}


$>$ Las Redes sociales de Internet constituyen un medio eficaz para la comunicación y promoción turística y son empleadas de forma exitosa por pueblos turísticos en diversas partes del mundo.

$>$ En la actualidad el trabajo con las Redes sociales de Internet en pueblos turísitcos cubanos se encuentra limitado por problemas de infraestructura tecnológicas en las comunidades y falta de capacitación de los residentes en temas relativos a la comunicación promocional por Redes Sociales de Internet.

$>$ Los pueblos turísticos cubanos no son gestionados desde el uso delas redes sociales de Internet, dejando su imagen en manos de otras personas o instituciones quienes la utilizan en correspondencia a intereses propios.

Las acciones propuestas a partir del uso de las redes sociales de Internet permiten aprovechar las ventajas de las mismas para comunicar los valores históricosculturales-naturales de los pueblos turísticos cubanos.

\section{Referencias Bibliográficas}

SALGADO FLEBES, José Comercio Electrónico y Turismo. Ministerio del Turismo de la República de Cuba Centro de Estudios Turísticos de la Universidad de la Habana.Ed:Félix Varela .2009. p. 115. ISBN: 978-959-07-1129-9

CUBA GONZÁLEZ, Yalín Santa María del Rosario: Propuesta como pueblo de Interés Turístico. Trabajo de Diploma en Opción al título de Licenciado en Turismo. Facultad de Turismo. Universidad de la Habana. 2014.

CRUZ MARTÍNEZ, Gonazalo. Turismo y Políticas Públicas, análisis del programa de pueblos turísticos y su implementación en Carlos Keen. Argentina.Universidad Nacional de Mar del Plata. En: I Congreso Latinoamericano de Investigación Turística.(2014: Neuquén)

PARTIDO COMUNISTA DE CUBA. Lineamientos de la política económica y social del Partido y la Revolución. 2016.

LAFUENTE IBÁÑEZ, Carmen. Metodología de la Investigación en las Ciencias Sociales. Revista EAN (en linea) 64 (5-18) 2008. (Consulta: 10 enero 2018) Disponible en http://www.redalyc.org/pdf/206/20612981002.pdf. ISSN: 01208160.

QUESADARUIZ, Susana. Acciones estratégicas para la gestión de redes sociales de Internet en la División Cuba de Meliá Hoteles International para el periodo 20162017.Trabajo de Diploma en Opción al título de Licenciado en Turismo. Facultad de Turismo. Universidad de la Habana. 2016. 
EMPRESA DE TELECOMUNICACIONES DE CUBA “Confirma Etecsa avances en informatización de sociedad cubana”.2018.Disponible enwww.juventudrebelde.cu/cuba/2018-04-11/confirma-etecsa- avances-eninformatización-de-sociedad-cubanaISSN 1563-8340.

BAS VEN,Beld. Case Study of how a little town call Obermutten won Facebook. State of Digital Case of Study. 2013. (Consulta: 13 marzo 2018) Disponible en https://www.stateofdigital.com/case-study-facebook-engagement-a-little-towncalled-obermutten/

ORTEGA MALDONADO, Alvaro. La profesionalización del community manager.Revista Lasallista de Investigación (en linea) 14(217-232), 2017. (Consulta: 15 de marzo de 2018). Disponible en http://www.redalyc.org/pdf/695/69551301020.pdfISSN: 1794-4449

PEREZ CURIEL, Concha, LUQUE ORTIZ,Sergio El marketing de influencia en moda. Estudio del nuevo modelo de consumo en Instagram de los millennials universitarios. Revista Ad Comunica (en linea) 2018. (consulta: 18 de abril de 2018)

Disponible en http://www.adcomunicarevista.com/ojs/index.php/adcomunica/article/view/445/ 411.10(7-16) ISSN: 2254-2728

DUVAL SEGUÍN, Carlos. El Marketing Turístico 2.0 en las cadenas hoteleras de Cuba. Trabajo de Diploma en Opción al Título de Licenciado en Turismo. Facultad de Turismo. Universidad de la Habana La Habana. Cuba.2011.

LÓPEZ LEÓN, Delio. Estrategia para la Gestión de la Innovación en la Gerencia de Hoteles Habaguanex. Tesis de diploma en opción al título de Licenciada en Turismo. Facultad de Turismo. Universidad de La Habana. La Habana. Cuba.2014.

Sitios Web Consultados:www.consumerbarometer.com, www.internetworldstats.com. www.puebla.travel, www.mutten.ch, www.morellaturistica.com,www.statista.com. Redes sociales empeadas: Facebook, Twitter, YouTube, Instagram. 


\section{Para citar el artículo indexado.}

Martínez M., Robert M. \& Torres L. (2018). Las Redes Sociales de Internet para la promoción de pueblos turísticos. Revista electrónica Explorador Digital 2(2), 36-46. Recuperado desde:

http://cienciadigital.org/revistacienciadigital2/index.php/exploradordigital/article/view/331/7 $\underline{43}$

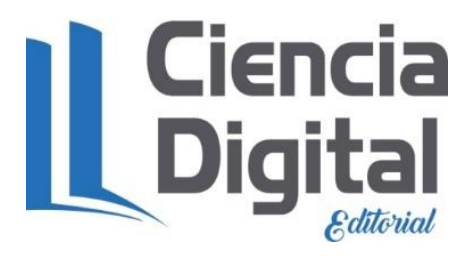

El artículo que se publica es de exclusiva responsabilidad de los autores y no necesariamente reflejan el pensamiento de la Revista Explorador Digital.

El articulo queda en propiedad de la revista y, por tanto, su publicación parcial y/o total en otro medio tiene que ser autorizado por el director o editor de la Revista Explorador Digital.
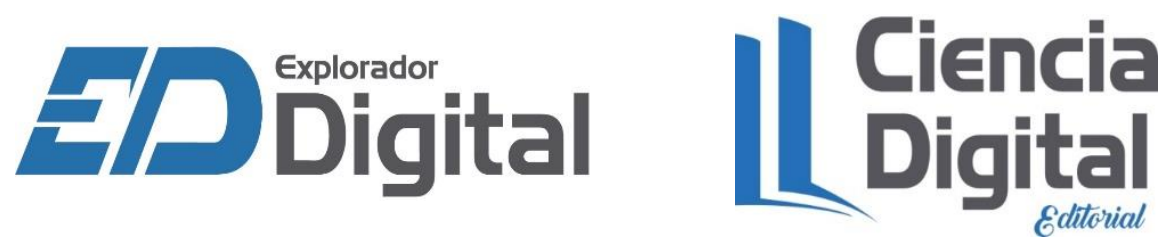